reduced bone mineral density (BMD), which increases risk of osteoporosis and fracture; however, there are currently no guidelines for the diagnosis or management of ADT-related osteoporosis. Alibhai and colleagues' study clarifies current Canadian clinical practice and perception of ADT-related osteoporosis risk.

A survey was mailed to all 228 active urologists in Ontario, plus 92 radiation oncologists who treat prostate cancer throughout Canada. Among these, 294 were prostate-cancer specialists of whom 172 responded. The 170 usable responses comprised data from 110 urologists and 60 radiation oncologists (overall response rate $58 \%$ ).

Radiation oncologists were more likely than urologists to assess baseline BMD routinely before initiating ADT ( $28 \%$ vs $5 \%$ ), but most ( $84 \%$ of radiation oncologists and $72 \%$ of urologists) would measure baseline BMD in patients with previous fractures. Weight-bearing exercise, avoidance of alcohol and cigarettes, and calcium and vitamin $D_{3}$ supplementation were most often recommended to treat or prevent ADT-related osteoporosis; by contrast, bisphosphonates were rarely recommended for prevention, although about half of physicians prescribed them for men starting ADT who already had osteoporosis. Respondents markedly overestimated the prevalence of osteoporosis. Physicians reported wide variation in the perceived risk of developing osteoporosis after 1 year of ADT.

Alibhai et al. highlight the large variation in screening methods for, and monitoring and treatment of, ADT-related osteoporosis. Educational efforts that target clinicians (especially those with few ADT patients, who were least likely to assess baseline BMD) are needed.

Original article Alibhai SMH et al. (2006) Prevention and management of osteoporosis in men receiving androgen deprivation therapy: a survey of urologists and radiation oncologists. Urology 68: 126-131

\section{Many cancer survivors bothered by ED have never tried therapy}

Treatments for localized prostate cancer commonly cause erectile dysfunction (ED), and about half of prostate cancer survivors seek therapy for ED within 5 years. Several studies have reported that surgically treated men are more likely to seek ED therapy than are radiotherapy-treated men; little is known, however, about how patients' concerns about (or indifference to) ED influences their use of ED therapies. Surprisingly, Miller et al. found that about half of radiotherapy-treated men had never tried ED therapies, despite reporting concern about their erectile function.

The authors analyzed responses to the Expanded Prostate Cancer Index Composite questionnaire, from 112 age-matched control individuals and 650 prostate cancer survivors, 4-8 years after treatment (radical prostatectomy, brachytherapy, or three-dimensional conformal external-beam radiotherapy). All treatment groups reported similar-quality unassisted erections (i.e. without use of ED therapies). Use of ED therapies was more common among cancer survivors than among controls $(30 \%$ versus $13 \%)$. About half of the prostate cancer survivors reported indifference (no concerns, despite poor or absent unassisted erections).

The authors note that radical prostatectomy causes immediate ED that gradually improves with time, whereas radiotherapy causes more gradual declines in erectile function that might not prompt patients to seek or accept ED therapy, and might not prompt clinicians to recommend the full range of ED therapies. Miller et al. call for additional studies to determine whether radiotherapy-treated patients tolerate their declines in erectile function, or have an unmet need for ED therapies.

Original article Miller DC et al. (2006) Use of medications or devices for erectile dysfunction among long-term prostate cancer treatment survivors: potential influence of sexual motivation and/or indifference. Urology 68: 166-171

\section{Tumor-invasion pattern: a new prognostic factor in penile carcinoma?}

Lymph-node metastasis is the most important prognostic factor in penile carcinoma, but cannot be reliably detected by clinical examination or imaging. Consequently, many of the lymphadenectomies that are routinely performed during surgical resection of penile carcinoma might be unnecessary. Guimarães and colleagues have identified the histologic pattern of tumor invasion into surrounding tissues as a potentially useful new prognostic factor in this condition.

They retrospectively reviewed clinical records and tissue samples from 112 men 